\title{
Força Muscular Respiratória e Capacidade Funcional na Insuficiência Renal Terminal
}

\author{
Respiratory Muscle Strength and Functional Capacity in End-stage \\ Renal Disease (ESRD)
}

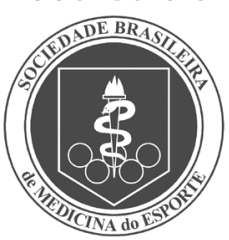

Artigo Original

Thiago Dipp ${ }^{1}$

Antônio Marcos Vargas da Silva²

Luis Ulisses Signori ${ }^{3}$

Tássio Müller Strimban ${ }^{4}$

Graziela Nicolodi ${ }^{4}$

Graciele Sbruzzi

Paulo Ricardo Moreira ${ }^{4}$

Rodrigo Della Méa Plentz ${ }^{1,5}$

1. Programa de Pós-Graduação em Ciências da Saúde: Cardiologia, Instituto de Cardiologia do Rio Grande do Sul/Fundação Universitária de Cardiologia - IC/ FUC, Porto Alegre, RS.

2. Departamento de Fisioterapia e Reabilitação, Universidade Federal de Santa Maria - UFSM, Santa Maria, RS. 3. Departamento de Ciências Biológicas, Universidade Federal de Rio Grande - FURG, Rio Grande, RS. 4. Departamento de Fisioterapia, Universidade de Cruz Alta - Unicruz, Cruz Alta, RS.

5. Departamento de Fisioterapia, Universidade Federal de Ciências da Saúde de Porto Alegre - UFCSPA,

Porto Alegre, RS.

\section{Endereço para correspondência:} Unidade de Pesquisa do IC/FUC Prof. Rodrigo Della Méa Plentz Av. Princesa Isabel 370 - Bairro Santana - 90620-000 - Porto Alegre, RS

E-mail: pesquisa.plentz@gmail.com roplentz@yahoo.com.br

\begin{abstract}
RESUMO
Objetivo: Verificar a associação da força muscular respiratória com a capacidade funcional, força proximal de membros inferiores e variáveis bioquímicas em pacientes em hemodiálise (HD). Métodos: Participaram deste estudo 30 indivíduos (18 homens), com 53,4 \pm 12,9 anos e tempo de HD de 41,1 $\pm 55,7$ meses. Foram avaliados pressão inspiratória máxima (PImax), pressão expiratória máxima (PEmax), distância percorrida no teste de caminhada de seis minutos (6MWT), número de repetições no teste de sentar-e-levantar em 30 segundos (TSL) e registrados os exames bioquímicos de rotina no serviço. Resultados: Houve diminuição da PEmax em relação aos valores preditos $(p=0,015)$ e redução na distância percorrida no $6 \mathrm{MWT}$ quando comparados com equações de predição ( $p<0,001)$. O logPImax e o logPEmax correlacionaram-se com o número de repetições no TSL ( $r=$ $0,476, p=0,008 ; r=0,540, p=0,002$, respectivamente), e com os níveis séricos de fósforo ( $r=0,422, p=0,020$; $r=0,639, p<0,001$, respectivamente). A distância no 6MWT correlacionou-se com o logPEmax ( $r=0,511 ; p=$ $0,004)$ e com o número de repetições no TSL $(r=0,561 ; p=0,001)$. Conclusão: A redução da PEmax em pacientes com IRT em HD está associada à capacidade funcional, força proximal de membros inferiores e níveis de fósforo sérico, podendo representar, pelo menos em parte, o baixo desempenho físico-funcional desses pacientes.
\end{abstract}

Palavras-chave: músculos respiratórios, insuficiência renal crônica, hemodiálise.

\begin{abstract}
Objective: to evaluate the association of respiratory muscle strength with functional capacity, lower limb strength and biochemical variables in hemodialysis (HD) patients. Methods: a cross-sectional study involving 30 patients (18 male), $53.4 \pm 12.9$ years, $41.1 \pm 55.7$ months on HD therapy. Maximal inspiratory pressure (PImax), maximal expiratory pressure (PEmax), distance completed in a six-minute walk test (6MWT) and number of repetitions in sit-and-stand test (STST) were evaluated. The biochemical variables were recorded in the database routine work service. Results: LogEPmax and 6MWT values were significantly lower than the predicted values $(p=0.015 ; p<0.001$, respectively). logPImax and logPEmax were correlated with number of repetitions in STST $(r=0.476, p=0.008 ; r=0.540, p=0.002$, respectively) and with phosphorus blood levels $(r=0.422, p=$ $0.020 ; r=0.639, p<0.001$, respectively). 6MWT was correlated with logPEmax $(r=0.511 ; p=0.004)$ and with number of repetitions in STST $(r=0.561 ; p=0.001)$. Conclusion: PEmax reduction in patients with ESRD on $\mathrm{HD}$ is associated with functional capacity, lower limb strength and phosphorus blood levels, and may at least partly represent the low physical and functional performance of these patients.
\end{abstract}

Keywords: respiratory muscles, chronic kidney failure, renal dialysis.

\section{INTRODUÇÃO}

A insuficiência renal crônica consiste em lesão renal e perda progressiva e irreversível da função dos rins, que em sua fase mais avançada é chamada de insuficiência renal terminal $(I R T)^{(1,2)}$, na qual se faz necessária alguma forma de terapia de substituição renal, sendo a hemodiálise (HD) a mais comum, com 66.833 pacientes em tratamento nos centros de diálise no Brasil ${ }^{(3,4)}$.

Pacientes com IRT sofrem de um conjunto de alterações em vários sistemas orgânicos, denominado de "síndrome urêmica"(5), o qual envolve uremia, anemia, doenças cardiopulmonares ${ }^{(6)}$, alterações metabólicas, disfunção musculoesquelética (miopatia urêmica) (7), comprometimento físico, imunológico e psíquico ${ }^{(5,8)}$. Esse fenômeno compromete a qualidade de vida e limita as atividades de vida diária, com aumento da taxa de mortalidade nessa população(9-11). A redução das medidas de pressão inspiratória e expiratória máxima ${ }^{(8)}$, representando a fraqueza muscular respiratória em pacientes com IRT, está associada à redução da capacidade funcional $\left.\right|^{(11,12)}$ e da qualidade de vida ${ }^{(13-15)}$.
Atualmente, grande interesse vem sendo atribuído à avaliação da capacidade funcional de pacientes com IRT através do teste de caminhada de seis minutos $(6 \mathrm{MWT})^{(16)}$, considerado um teste submáximo, com boa aceitação e exequibilidade(17), além de seu bom valor prognóstico ${ }^{(18)}$. A avaliação da força dos membros inferiores vem sendo realizada através do teste de sentar-e-levantar em 30 segundos (TSL) que já demonstrou ser uma mensuração válida e confiável da força proximal de membros inferiores ${ }^{(19,20)}$. Esses testes são simples, mensuram a capacidade funcional por meio de informações básicas, são de fácil aplicação, bem tolerados, não necessitam de equipamentos especiais além de serem representativos das atividades de vida diária ${ }^{(17,21)}$.

Recentes estudos demonstraram que um pobre controle do metabolismo mineral e do balanço de eletrólitos sanguíneos é um fator de risco independente para doença cardiovascular e para a mortalidade em pacientes em hemodiálise ${ }^{(22,23)}$. No entanto, a relação da força muscular respiratória com variáveis bioquímicas carece de maior elucidação em pacientes com IRT. 
Em se tratando de uma população que sofre com as alterações fisiopatológicas sistêmicas inerentes à IRT, inclusive a redução da força muscular respiratória e capacidade funcional, o objetivo desse trabalho foi investigar a associação da força muscular respiratória com variáveis funcionais e bioquímicas em pacientes com IRT.

\section{MÉTODOS}

Esse estudo do tipo transversal foi constituído por uma amostra consecutiva de 30 pacientes portadores de IRT submetidos a três sessões semanais de HD, com duração entre três e quatro horas/sessão, na Clínica Renal Santa Lúcia/Cruz Alta - RS.

As medicações e comorbidades foram recordados com base nos três últimos meses através dos prontuários individuais da própria clínica, e os exames bioquímicos foram realizados no dia da coleta dos exames funcionais. Foram incluídos sujeitos adultos, de ambos os sexos, em tratamento dialítico por período mínimo de seis meses. Foram excluídos pacientes com angina instável, arritmias cardíacas graves, insuficiência cardíaca descompensada, hipertensão arterial não controlada (pressão arterial sistólica $>200 \mathrm{mmHg}$ e pressão arterial diastólica $>120 \mathrm{mmHg}$ ), diabetes melito descompensada (glicemia $>300 \mathrm{mg} / \mathrm{dL}$ ), pneumopatia crônica, infecção sistêmica aguda, osteodistrofia renal grave, distúrbios neurológicos, musculoesqueléticos e osteoarticulares incapacitantes. O projeto foi aprovado pelo Comitê de Ética em Pesquisa de nossas instituições e todos os voluntários leram e assinaram o Termo de Consentimento Livre e Esclarecido. Todas as avaliações a seguir foram realizadas antes da segunda sessão semanal de hemodiálise.

A força muscular respiratória foi avaliada pela medida das pressões inspiratórias (PImax) e expiratórias máximas (PEmax), a partir do volume residual e da capacidade pulmonar total, respectivamente, com um manovacuômetro (Famabras, Indústria Brasileira) escalonado em -300 a $+300 \mathrm{cmH}_{2} \mathrm{O}$, de acordo com metodologia proposta por Black e Hyatt(24). Os pacientes foram instruídos a realizar três esforços máximos durante um segundo, contra uma via ocluída com um pequeno orifício de escape para prevenir que os pacientes mantivessem a glote aberta, evitando a ação dos músculos da boca. Foram realizadas três manobras com o uso de um clipe nasal, sendo considerada a média entre elas e calculados os valores preditos pelas equações propostas por Neder et al.(24).

A capacidade funcional foi avaliada pelo 6MWT de acordo com as diretrizes propostas pela American Thoracic Society ${ }^{(25)}$. Foram monitoradas a pressão arterial, frequência cardíaca e o nível de dispneia (escala de percepção de esforço de Borg) antes e após os testes. Foram usadas as equações propostas por Enright e Sherrill(26) para predição da distância percorrida no 6MWT.

A avaliação da força e resistência dos membros inferiores foi realizada através do teste de sentar-e-levantar em 30seg (TSL) com o paciente sentado em uma cadeira com $45 \mathrm{~cm}$ de altura, com as costas eretas e os pés afastados na largura dos ombros e apoiados no solo ${ }^{(19)}$. Os braços foram cruzados ao nível dos punhos e contra o peito. Ao sinal de partida o sujeito tinha que se levantar até ficar em pé regressando à posição sentada em um intervalo de 30seg, sendo registrado o número máximo de repetições.

\section{Análise estatística}

Os dados foram analisados no programa Statistical Package for the Social Sciences (SPSS) versão 13.0 para Windows e estão expressos como média \pm desvio padrão. O teste de Kolmogorov-Smirnov foi utilizado para verificar a distribuição dos dados e realizada a transformação logarítmica para as variáveis com distribuição assimétrica. Para comparação das médias dos valores obtidos e preditos foi utilizado o teste $t$ de Student emparelhado. Para verificar a associação entre as variáveis de força muscular respiratória e as variáveis funcionais e bioquímicas, foi utilizado o coeficiente de correlação de Pearson. Análise de regressão multivariada foi utilizada para avaliar o impacto de potenciais determinantes nas variáveis funcionais. Foi considerado um nível de significância de 5\% ( $p<0,05)$.

\section{RESULTADOS}

Foram avaliados 30 indivíduos com média de idade de 53,4 \pm 12,9 anos (variando de 30 a 82 anos), sendo 18 homens e 12 mulheres. Quanto ao índice de massa corporal (IMC) 40\% ( $n=12$ ) foram classificados como eutróficos, 33\% ( $n=10$ ) apresentaram sobrepeso e 27\% ( $n=8$ ) obesidade. 0 tempo médio de tratamento hemodialítico foi de 41,1 \pm 55,7 meses (variando de seis a 273 meses). A hipercolesterolemia foi observada em 33\% dos voluntários, hiperglicemia em 13\%, hipertrigliceridemia em 50\%, hipocalcemia em 50\%, hiperfosfatemia em 36,6\% e hiperpotassemia em 60\% da amostra (tabela 1).

Tabela 1. Características demográficas e laboratoriais dos pacientes.

\begin{tabular}{|c|c|c|}
\hline & Média (n = 30) & $D P(n=30)$ \\
\hline Idade (anos) & 53,4 & 12,9 \\
\hline Peso (kg) & 71,6 & 16,7 \\
\hline $\mathrm{IMC}\left(\mathrm{kg} / \mathrm{m}^{2}\right)$ & 26,5 & 5,2 \\
\hline Tempo diálise (meses) & 41,1 & 55,7 \\
\hline Colesterol (mg/dL) & 174,7 & 39,7 \\
\hline Glicose (mg/dL) & 82,8 & 23,8 \\
\hline Triglicerídeos (mg/dL) & 206,7 & 140,6 \\
\hline Cálcio (mg/dL) & 8,7 & 0,7 \\
\hline Fósforo (mg/dL) & 4,4 & 1,2 \\
\hline Ferritina (mg/mL) & 458,4 & 205,1 \\
\hline Ferro sérico (mg/dL) & 73,2 & 30,1 \\
\hline Fosfatase alcalina (U/L) & 149,6 & 150,7 \\
\hline Potássio (mmol/L) & 5,4 & 0,6 \\
\hline Albumina (g/dL) & 3,9 & 0,3 \\
\hline Creatinina (mg/dL) & 8,8 & 3,6 \\
\hline Proteínas (g/dL) & 6,8 & 0,6 \\
\hline $\mathrm{Kt} / \mathrm{N}$ & 1,30 & 0,3 \\
\hline Hemoglobina (g/dL) & 10,9 & 2,1 \\
\hline Hematócrito (\%) & 34,1 & 6,6 \\
\hline Leucócitos $\left(\times 10^{3} / \mathrm{mm}^{3}\right)$ & 6.300 & 1.445 \\
\hline
\end{tabular}

O valor médio do índice de eficácia da diálise (Kt/N) foi igual a 1,30 $\pm 0,3$. A média dos níveis de hemoglobina foi de 10,9 $\pm 2,1 \mathrm{~g} / \mathrm{dL}$ (variando de 6,6 a 16,3) com 43,3\% apresentando níveis inferiores a $11 \mathrm{mg} /$ $\mathrm{dL}$ (tabela 1). A eritropoetina recombinante humana foi utilizada em $63,7 \%$ dos pacientes (9.333 $\pm 2.808 \mathrm{U} /$ semana), antianêmico em 87\%, suplementação com cálcio em $80 \%$, inibidor da enzima conversora de angiotensina em 27\%, estatina em 57\%, ácido acetilsalicílico em 47\% e hipoglicemiante em $17 \%$ dos pacientes.

Com relação às pressões respiratórias máximas obtidas e preditas, a amostra demonstrou redução significativa da PEmax de 14,2\% (91 \pm 48,7 vs. 106,1 $\pm 19,5 \mathrm{cmH}_{2} \mathrm{O} ; \mathrm{p}=0,015$ ) em relação aos valores preditos. Entretanto, a PImax não apresentou diferença entre os valores obtidos e preditos $(99,7 \pm 55,8$ vs. 100,7 $\pm 15,1 ; p=n s)$.

Dados relativos às avaliações funcionais mostraram que no 6MWT houve redução significativa de 27,7\% (397 \pm 104 vs. 548,7 \pm 104,1 m; p =0,001) entre a distância obtida e a distância predita pelas equações. A distância obtida variou de $120 \mathrm{~m}$ a $600 \mathrm{~m}$ e a prevista entre $226 \mathrm{~m}$ e $707 \mathrm{~m}$. No TSL o número de repetições variou de sete a 18.

O logPImax correlacionou-se diretamente com oTSL $(r=0,456 ; p=$ $0,008$; figura $1 \mathrm{~A})$ e com os níveis plasmáticos de fósforo $(r=0,422 ; p=$ $0,020)$. O logPEmax também esteve correlacionado com TSL ( $r=0,540$; $p=0,002$; figura 1B) e níveis de fósforo $(r=0,639 ; p<0,001)$. A distância percorrida no 6MWT correlacionou-se com o logPEmax $(r=0,511 ; p=$ 0,004; figura 2A) e com o número de repetições no TSL $(r=0,561 ; p=$ 0,001; figura 2B), porém, não foi evidenciada correlação significativa do 


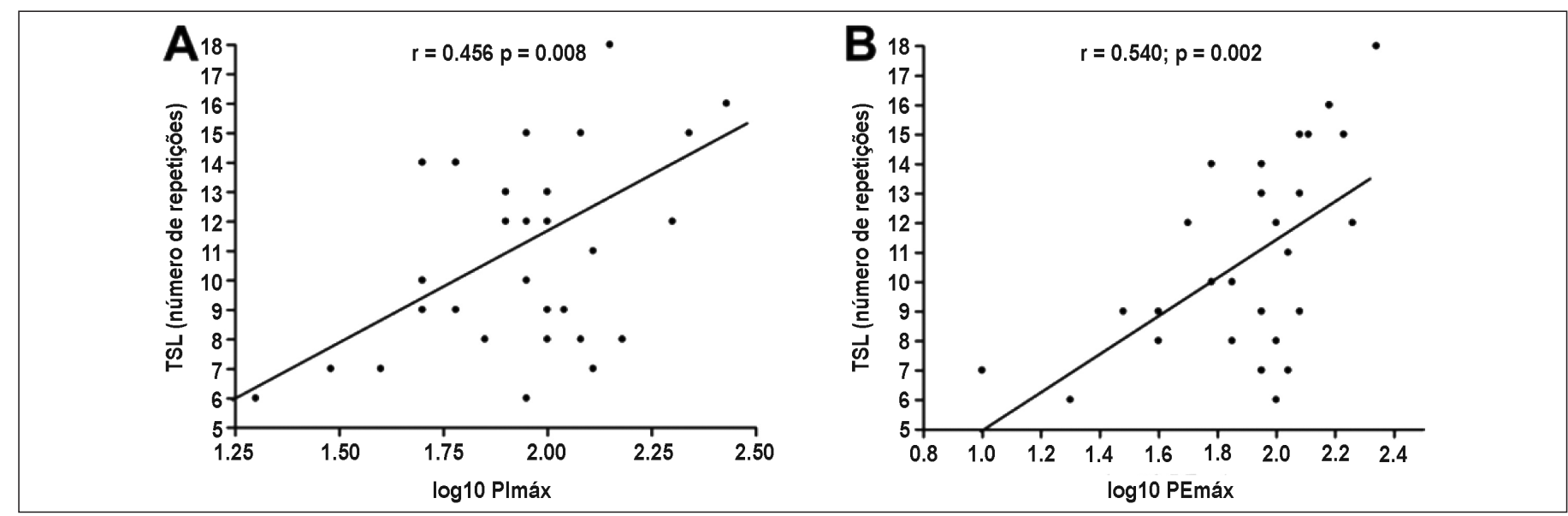

Figura 1. Correlação do TSL com pressões respiratórias máximas: (A) TSL x logPIMax (B) TSL x logPEMax.

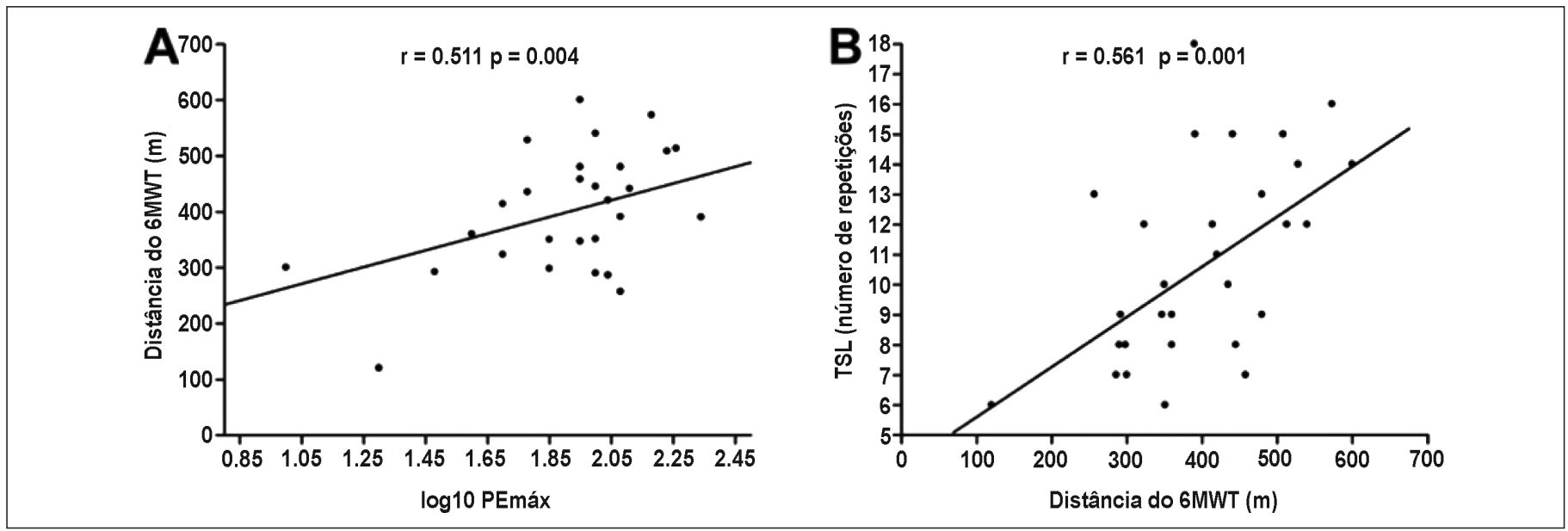

Figura 2. Correlação do 6MWT com pressão expiratória máxima e TSL: (A) 6MWT x logPEMax (B) TSL x 6MWT.

6MWT com o logPImax ( $r=0,345 ; p=0,62)$. A idade correlacionou-se inversamente com a distância percorrida no $6 \mathrm{MWT}(r=-0,465 ; p=0,010)$ e com o número de repetições noTSL $(r=-0,508 ; p=0,004)$. Não foram identificadas correlações significativas do logPImax e do logPEmax com a idade. A força muscular respiratória e as variáveis funcionais (TSL e 6MWT) não apresentaram correlação com o peso, IMC, tempo de diálise, cálcio, ferro sérico, potássio, creatinina, hemoglobina e hematócrito.

Na análise da regressão linear múltipla usando o TSL como variável dependente, a idade e PImax foram responsáveis por 46,9\% $\left(R^{2}=0,469\right)$ da variação do número de repetições. Para cada ano de vida houve diminuição de 0,126 ( $p=0,001)$ repetições, enquanto que para cada unidade de aumento na PImax houve elevação de 0,027 ( $p=0,003)$ repetições. Quando substituída a PImax pela PEmax, idade e PEmax explicaram 47,6\% $\left(R^{2}=0,476\right)$ da variação do número de repetições. Para cada ano de vida houve diminuição de 0,085 repetições ( $p=$ 0,031 ) enquanto que para cada unidade de aumento de PEmax o TSL aumentou 0,033 ( $p=0,002)$ repetições.

\section{DISCUSSÃO}

Os pacientes apresentaram redução da PEmax e da distância percorrida no 6MWT em relação aos valores preditos, representando, respectivamente, fraqueza muscular expiratória e diminuição da capacidade funcional. O nosso estudo demonstrou pela primeira vez a associação da força muscular respiratória com níveis de fósforo circulantes, força muscular proximal de membros inferiores e capacidade funcional em pacientes com IRT em HD.

As manifestações da síndrome urêmica afetam negativamente a capacidade funcional(12,27,28), a qualidade de vida ${ }^{(13-15)}$, a força muscular respiratória(12,13,29) e função pulmonar ${ }^{(30,31)}$, fato que pode estar associado à diminuição da força muscular periférica evidenciada em pacientes com $\mathrm{IRT}^{(9,32)}$. Assim como em outros trabalhos ${ }^{(8,13)}$, os pacientes apresentaram redução da PEmax em relação ao previsto pelas equações de Neder et al. ${ }^{(24)}$. Esse achado pode ser explicado pela ocorrência da miopatia urêmica que altera intensamente o sistema muscular esquelético. No entanto, a média da PImax na amostra não diferiu dos valores previstos ${ }^{(24)}$ e, dessa forma, pode representar que a força muscular inspiratória está preservada em pacientes em HD.

Também foi verificada nos pacientes redução da distância percorrida no 6MWT em relação ao previsto pelas equações de Enright e Sherrill(26) para indivíduos normais. O 6MWT foi o teste de escolha para estimar a capacidade funcional em virtude de apresentar boa aplicabilidade em pacientes com IRT, além de ser considerado teste submáximo capaz de refletir a aptidão cardiorrespiratória( ${ }^{(16)}$. Resultados semelhantes foram descritos no estudo de Hsieh et al. ${ }^{(33)}$, envolvendo 27 pacientes com IRT submetidos à HD, no qual relatou que a distância obtida no 6MWT (398,8 \pm 74,5m) correlacionou-se positivamente com o consumo máximo de oxigênio $\left(\mathrm{VO}_{2}\right.$ máx) $(11,5 \pm 2,9 \mathrm{~mL} / \mathrm{kg} / \mathrm{min} ; \mathrm{r}=0,62)$. Embora a média de idade da amostra desse estudo tenha sido menor que a do estudo anterior ${ }^{(33)}$, os pacientes percorreram uma distância semelhante. Da mesma forma, Cury et al..(12) encontraram valores de distância percorrida no 6MWT menores que 500m na maioria dos pacientes em HD da sua amostra. Em contrapartida, distâncias maiores $(516 \pm 88,79 m)$ foram demonstradas por Reboredo et al. ${ }^{(16)}$, que também referiram correlação positiva $(r=0,78)$ com o VO 2 máx em 16 pacientes em HD. Essas diferenças na performance durante o 6MWT podem ser atribuídas à grande heterogeneidade nas características fisiopatológicas, clínicas, terapêuticas e físico-funcionais identificadas nessa população. 
Quanto à força muscular proximal de membros inferiores, não foram encontrados valores de referência na literatura ao utilizar o TSL como método de avaliação, provavelmente pelas discrepâncias metodológicas na utilização do teste. Alguns autores aplicaram o TSL tendo como variável mensurada o tempo para fadiga ${ }^{(9)}$, o número máximo de repetições em um minuto ${ }^{(9)}$ ou em 30seg ${ }^{(19)}$. No presente trabalho adotou-se a medida do número máximo de repetições em 30seg em virtude das debilidades físico-funcionais particulares dos pacientes com IRT. Esse trabalho demonstrou que a PImax e PEmax se correlacionam diretamente com o TSL, podendo ser um indicativo de que a miopatia urêmica afeta de forma paralela os músculos respiratórios e de membros inferiores de pacientes em HD. Além disso, houve associação mais forte entre PEmax eTSL, o que pode representar relação mais pronunciada dos músculos expiratórios com os grupos musculares proximais dos membros inferiores.

Pacientes com IRT estão expostos a constantes alterações bioquímicas e eletrolíticas ${ }^{(22,34)}$ que podem estar relacionadas com baixos níveis de capacidade física ${ }^{(10)}$ e, dessa maneira, influenciar na contração muscular e na capacidade de realização de exercício. Foi possível observar que $36,6 \%$ dos pacientes estudados apresentaram hiperfosfatemia, sendo que o fósforo sérico foi a única variável bioquímica que esteve associada com a força muscular respiratória, com forte correlação com a PEmax ( $r$ $=0,63)$ e moderada com a PImax $(r=0,42)$. Ulubay et al. ${ }^{(10)}$, investigando possíveis fatores que influenciavam na aptidão cardiorrespiratória em pacientes tratados com diálise peritoneal pré-transplante renal, demonstraram correlação moderada do $\mathrm{VO}_{2}$ máx com níveis séricos de fósforo $(r=0,59)$ e duração do teste ergoespirométrico $(r=0,53)$. A associação da força muscular respiratória e periférica com níveis de fósforo já foi relatada por Fiaccadori et al. ${ }^{(35)}$ em sujeitos com doença pulmonar obstrutiva crônica, avaliada através de biópsia muscular de quadríceps e intercostal externo. O fósforo é um elemento essencial em diversos processos envolvidos na produção, transporte e utilização da energia celular, como a contração muscular esquelética ${ }^{(10)}$. Esses achados seguem a mesma tendência dos resultados desse trabalho, podendo indicar que a fraqueza dos músculos respiratórios está relacionada a distúrbios do metabolismo do fósforo. Porém, ainda são necessários maiores estudos para avaliar os efeitos do controle nos níveis de fósforo circulante sobre a função muscular respiratória de pacientes com IRT.

\section{REFERÊNCIAS}

1. Romão Jr J. Renal Crônica: Definição, Epidemiologia e Classificação. J Bras Nefrol 2004;26:1-3.

2. Lang $S M$, et al. Acute effects of hemodialysis on lung function in patients with end-stage renal disease. Wien Klin Wochenschr 2006;118:108-13.

3. Kopple JD, Storer T, Casburi R. Impaired exercise capacity and exercise training in maintenance hemodialysis patients. J Ren Nutr 2005;15:44-8.

4. Sesso R, et al. Resultados do Censo de Diálise da SBN 2007. J Bras Nefrol 2007;29:197-202.

5. Johansen KL. Physical functioning and exercise capacity in patients on dialysis. Adv Ren Replace Ther 1999:6:141-8

6. Ammirati AL, Canziani MEF. Fatores de risco para doença cardiovascular nos pacientes com doença renal crônica. J Bras Nefrol 2009;31:43-8.

7. Carrero JJ, et al. Muscle atrophy, inflammation and clinical outcome in incident and prevalent dialysis patients. Clin Nutr 2008:27:557-64.

8. Karacan $\mathrm{O}$, et al. Pulmonary function in renal transplant recipients and end-stage renal disease patients undergoing maintenance dialysis. Transplant Proc. 2006;38:396-400.

9. McIntyre CW, et al. Patients receiving maintenance dialysis have more severe functionally significant skeletal muscle wasting than patients with dialysis-independent chronic kidney disease. Nephrol Dial Transplant 2006;21:2210-6.

10. Ulubay $G$, et al. Factors affecting exercise capacity in renal transplantation candidates on continuous ambulatory peritoneal dialysis therapy. Transplant Proc 2006;38:401-5.

11. Reid WD, Dechman G. Considerations when testing and training the respiratory muscles. Phys Ther 1995;75:971-82.

12. Cury JL, Brunetto AF, Aydos RD. Efeitos negativos da insuficiência renal crônica sobre a função pulmonar e a capacidade funcional. Rev Bras Fisioter 2010;14:91-8.

13. Schardong T, Lukrafka J, Garcia V. Avaliação da função pulmonar e da qualidade de vida em pacientes com doença renal crônica submetidos a hemodiálise. J Bras Nefrol 2008;30:40-7.

14. Santos R, Pontes $L$. Mudança do nível de qualidade de vida em portadores de insuficiência renal crônica terminal durante seguimento de 12 meses. Rev Assoc Med Bras 2007;53:329-34.

15. Barbosa L, Júnior M, Bastos K. Preditores de qualidade de vida em pacientes com doença renal crônica em hemodiálise. J Bras Nefrol 2007;29:222-9.

16. Reboredo $\mathrm{M}$, et al. Correlação entre a distância obtida no teste de caminhada de seis minutos e o pico de consumo de oxigênio em pacientes portadores de doença renal crônica em hemodiálise. J Bras Nefro 2007;29:83-7.

17. Solway S, et al. A qualitative systematic overview of the measurement properties of functional walk tests used in the cardiorespiratory domain. Chest 2001;119:256-70.

18. Sietsema KE, et al. Exercise capacity as a predictor of survival among ambulatory patients with end-stage renal disease. Kidney Int 2004;65:719-24.
A distância percorrida no 6MWT esteve correlacionada positivamente com PEmax e TSL, demonstrando o envolvimento da força dos músculos expiratórios e dos músculos proximais de membros inferiores no desempenho durante a caminhada ${ }^{(36)}$. Como a PImax não se correlacionou com o 6MWT, podemos inferir que os músculos expiratórios (abdominais, intercostais internos, esternocleidomastoideos etc), pelo seu grande papel na manutenção do equilíbrio ácido-básico com o aumento da demanda ventilatória, exercem maior influência durante o esforço em pacientes com IRT. Assim, o desempenho desses grupos musculares pode determinar, pelo menos em parte, a distância percorrida durante o 6MWT e o seu treinamento pode fazer parte das estratégias na reabilitação física para esses pacientes.

Na análise multivariada com o TSL como variável dependente, a idade, PImax e PEmax foram preditoras de mudanças na força proximal de membros inferiores. A idade combinada com a PImax ou com a PEmax foram capazes de explicar aproximadamente 50\% do desempenho dos pacientes durante o TSL, mostrando que os dois modelos apresentados, envolvendo a medida de força dos músculos inspiratórios e expiratórios, mostraram-se fortes preditores de fraqueza muscular de membros inferiores. Torna-se necessária a ampliação da amostra para viabilizar uma análise de regressão e, dessa forma, estabelecer uma relação causa-efeito entre idade, fraqueza muscular respiratória e periférica de membros inferiores em pacientes com IRT em HD.

\section{CONCLUSÃO}

O presente estudo demonstra importante associação da força muscular respiratória com capacidade funcional, força proximal de membros inferiores e níveis de fósforo sérico em pacientes em HD, podendo representar, pelo menos em parte, os baixos níveis de desempenho físico-funcional relatado nessa população. A força dos músculos expiratórios pode ser tão importante quanto à força muscular de membros inferiores em pacientes com IRT em HD, e podem fazer parte da rotina de avaliação e tratamento para a otimização do processo de reabilitação desses pacientes.

Todos os autores declararam não haver qualquer potencial conflito de interesses referente a este artigo.

19. Jones CJ, Rikli RE, Beam WC. A 30-s chair-stand test as a measure of lower body strength in community residing older adults. Res Q Exerc Sport. 1999;70:113-9.

20. Painter $P$, et al. Physical functioning and health-related quality-of-life changes with exercise training in hemodialysis patients. Am J Kidney Dis 2000;35:482-92.

21. Rosie J, Taylor D. Sit-to-stand as home exercise for mobility-limited adults over 80 years of age - GrandStand System may keep you standing? Age Ageing 2007;36:555-62.

22. Pourfarziani V, et al. Laboratory variables and treatment adequacy in hemodialysis patients in Iran. Saudi $J$ Kidney Dis Transpl 2008;19:842-6.

23. Phelan PJ, et al. The importance of serum albumin and phosphorous as predictors of mortality in ESRD patients. Ren Fail 2008:30:423-9.

24. Neder J, et al. Reference values for lung function tests II. Maximal respiratory pressures and voluntary ventilation. Braz J Med Biol Res 1999;32:719-27.

25. ATS statement: guidelines for the six-minute walk test. Am J Respir Crit Care Med 2002:166:111-7.

26. Enright PL, Sherrill DL. Reference equations for the six-minute walk in healthy adults. Am J Respir Crit Care Med 1998;158:1384-7.

27. Parsons TL, Toffelmire EB, King-VanVlack, CE. Exercise training during hemodialysis improves dialysis efficacy and physical performance. Arch Phys Med Rehabil 2006;87:680-7.

28. Van Laethem C, et al. Chronic kidney disease is associated with decreased exercise capacity and impaired ventilatory efficiency in heart transplantation patients. J Heart Lung Transplant 2009;28:446-52.

29. Kovelis $D$, et al. Funçăo pulmonar e força muscular respiratória em pacientes com doença renal crônica submetidos à hemodiálise. J Bras Pneumol 2008;34:907-12

30. Bavbek N, Akay H, Uz B, Uz E, Turgut F, Kanbay M, et al. The effects of L-carnitine therapy on respiratory function tests in chronic hemodialysis patients. Ren Fail 2010;32:157-61.

31. Yoon SH, Choi NW, Yun SR. Pulmonary dysfunction is possibly a marker of malnutrition and inflammation but not mortality in patients with end-stage renal disease. Nephron Clin Pract 2009;111:c1-6.

32. Padilla J, et al. Physical functioning in patients with chronic kidney disease. J Nephrol 2008;21:550-9.

33. Hsieh RL, Lee WC, Chang CH. Maximal cardiovascular fitness and its correlates in ambulatory hemodialysis patients. Am J Kidney Dis 2006;48:21-7.

34. Ferrari $\mathrm{P}$, et al. Serum phosphate is an important determinant of corrected serum calcium in end-stage kidney disease. Nephrology (Carlton) 2009;14:383-8.

35. Fiaccadori E, et al. Hypophosphatemia and phosphorus depletion in respiratory and peripheral muscles of patients with respiratory failure due to COPD. Chest 1994;105:1392-8.

36. Plentz RDM, et al. Correlaçăo entre o teste de caminhada de seis minutos e força muscular respiratória em IRC. Rev Bras Fisioter 2007,11:354-354. 